\title{
Revue
}

\section{Conduites de troupeaux ovins en extensif}

\author{
Benoît Dedieu ${ }^{a *}$, Jean-Marc Chabosseau ${ }^{\mathrm{b}}$, Marc Benoit $^{\mathrm{c}}$, \\ Gabriel Laignelc
}

\author{
${ }^{\text {a }}$ Inra, départements SAD et ENA, laboratoire adaptation des herbivores aux milieux, \\ 63122 Saint-Genès-Champanelle, France \\ ${ }^{b}$ Inra, station d'amélioration des plantes fourragères, 86500 Lusignan, France \\ c Inra, LEE, 63122 Saint-Genès-Champanelle, France
}

(Reçu le 24 avril 1998 ; accepté le 8 juillet 1998)

\begin{abstract}
Diversity of flock management in extensive conditions. Management practices in 13 extensively farmed sheep flocks (stocking level less than $1 \mathrm{LU} \cdot \mathrm{ha}^{-1}$ principal forage surface) in Montmorillonnais (a west-central region of France) were analyzed with attention to three components of the farming projects: autonomous foraging goals (breeding based on grass), attention to buyers' requests (records of first semester sales) and simplicity of flock management. Three different farming styles were identified that either limited the amount of concentrate used, encouraged sales early in the year, or that involved both of these factors. In the most autonomic systems, savings in concentrate concern either the ewes and the lambs. A simple farming style, indicated by a stable grouping of ewes, was observed in situations where the farmer was the only permanent employee (four out of six cases). The technical and economic performances (gross margin per ewe) were more or less equivalent whatever the management style or ewe grouping. Taking into account the spreading of lambing throughout the year, the different ways of grouping ewes and the changes in stocking level during the winter all helped to clarify the technical questions associated with extensive farming. (C) Elsevier / Inra
\end{abstract}

\section{extensive farming / sheep systems / flock management / breeding projects}

Résumé - Les pratiques de conduite de 13 troupeaux ovins extensifs (chargement inférieur à 1 UGB $\cdot$ ha $^{-1}$ SFP) du Montmorillonais (Centre Ouest) sont analysées en référence à trois déterminants des projets d'élevage : la recherche d'une autonomie fourragère (élevage basé sur l'herbe), la satisfaction de la demande de la filière (plus de ventes d'agneaux au premier semestre) et la simplicité de la conduite. Trois types de conduite sont identifiés, privilégiant l'économie de concentrés, des ventes précoces en début d'année, ou les deux à la fois. Les situations les plus autonomes renvoient à une recherche d'économie de concentrés portant à la fois sur l'alimentation des mères en plus de celui des agneaux. La simplicité de la conduite, repérée par une formule stable d'allotement des brebis, est

* Correspondance et tirés à part

Tél. : (33) 0473624038 ; fax : (33) 73624118 ; e-mail : dedieu@ clermont-inra.fr 
manifeste dans les situations où l'agriculteur est le seul permanent (quatre cas sur six). Les performances techniques et économiques (marge brute par brebis) sont sensiblement équivalentes selon les projets d'élevage. La prise en compte des répartition des mises bas dans l'année, des formules d'allotement et de l'ajustement du chargement aux disponibilités hivernales précisent les questions techniques associées à l'«extensif ». (1) Elsevier / Inra

élevage extensif / élevage ovin / conduite de troupeaux / projets d'élevage

\section{INTRODUCTION}

De nombreuses études ont été menées sur le fonctionnement des systèmes d'élevage extensif depuis la fin des années 1980 $[1,4,10,15,17,19]$. La plupart des auteurs s'accordent pour constater qu'un faible niveau de chargement n'est pas un objectif en soi mais plutôt la conséquence d'autres priorités techniques et économiques [18] voire la simple prolongation d'un contexte historique sur l'exploitation. Réduire les coûts de production, disposer d'une plus grande souplesse au niveau fourrager pour pouvoir gérer avec simplicité un grand troupeau, tirer parti d'espaces disponibles à faible coût, rechercher de la plus value sur des produits à l'image «naturelle » (élevage à base d'herbe), obtenir des subventions sont autant de raisons citées qui justifient ou sont associées au caractère extensif des élevages observés. La diversité des conduites d'élevage extensif, constatée dans ces études, s'expliquerait ainsi d'abord par la variabilité des projets d'élevage.

Notre objectif est d'analyser la diversité de conduites de troupeaux en conditions extensives, à partir des exploitations du réseau ovin extensif « Montmorillonnais » (Vienne, France). Dans cette région herbagère du Centre-Ouest, les choix de conduite dépendent de trois types de préoccupations : recherche d'une autonomie fourragère (élevage basé sur l'herbe), satisfaction de la demande des opérateurs d'aval (étalement des livraisons d'agneaux de boucherie), et simplicité de la conduite (composante de la maîtrise du travail).

\section{ORIGINE DES DONNÉES}

Les modalités de constitution du réseau ovin extensif Montmorillonnais, le protocole de suivi, et les caractéristiques générales des exploitations ont été précisés par ailleurs [5]. Treize exploitations, au chargement inférieur ou égal à $1 \mathrm{UGB} \cdot \mathrm{ha}^{-1}$ de SFP et ayant des troupeaux ovins ont été sélectionnées et suivies pendant trois ans (1993-1995). Seules les données de l'année 1994 sont présentées ici.

La collecte d'information s'est focalisée sur les pratiques d'élevage, de gestion des surfaces et l'établissement des résultats d'exploitations. Le support de collecte de l'ensemble des pratiques de conduite des troupeaux est le schéma d'allotement proposé par Ingrand et al. [12], sur lequel figure l'évolution au cours du temps des lots d'animaux (brebis, agneaux) gérés par l'éleveur.

L'analyse des données s'appuie sur la démarche et les concepts de l'approche systémique du fonctionnement des systèmes d'élevage [16]. À partir de la description et de la compréhension des pratiques de conduite, nous identifions les projets d'élevage. Puis nous envisageons l'effet de ces pratiques sur les résultats techniques et économiques.

\section{LES PRATIQUES D'ÉLEVAGE}

Les brebis sont toutes, à un élevage près, issues de croisements non fixés de races d'herbages (Vendéens, Texel, Charollais, Suffolk, Charmois...). Les mâles reproducteurs sont également de types génétiques variés. 


\subsection{La conduite de la reproduction}

Dans toutes les situations, des mises bas ont lieu entre février et avril. La date de début des mises bas d'hiver et l'existence ou non de mises bas d'automne permettent de distinguer trois types de conduites de la reproduction :

1) des agnelages en automne et en janvier (début des mises bas entre le $1^{\text {er }}$ et le 12 janvier). Six éleveurs adoptent cette conduite, avec plus d'un quart des mises bas réparties entre septembre et fin janvier (de 25 à $61 \%$ ). Les agneaux nés à ces périodes qu'ils soient engraissés en bergerie ou élevés à l'herbe (naissances de janvier) peuvent être commercialisés sans difficulté au premier semestre, période où les cours sont généralement les plus favorables ;

2) agnelages d'automne et d'hiver, ce dernier ne débutant qu'à partir du 25 janvier au plus tôt (au 1/02 en moyenne). Moins de $20 \%$ des mises bas totales ont lieu à l'automne (de 10 à 19\%). Ce type concerne cinq élevages ;

3) pas de contre-saison ni d'agnelages de début janvier: l'agnelage unique d'hiver débute fin janvier (un cas) et fin février (un cas).

\subsection{Hivernage et alimentation des brebis}

Les brebis agnelant à l'automne rentrent en bergerie à partir de la dernière quinzaine précédant la date présumée de début des mises bas et ne ressortent, d'une manière générale, qu'après le sevrage des agneaux (à deux mois et demi d'âge en moyenne). Les pratiques sont plus diversifiées en hiver. Seuls deux éleveurs, ayant moins de 450 brebis, hivernent l'ensemble de leurs brebis simultanément. À l'opposé, deux autres pratiquant le plein air ne rentrent les brebis que pour surveiller l'agnelage et la bonne adoption des agneaux (deux à quatre jours en moyenne). Pour le reste, la taille des bâtiments ne permet de rentrer qu'une partie du troupeau seulement. La durée de présence des brebis en bergerie est alors d'un à deux mois.

Du fait de la faible durée de l'hivernage, l'alimentation des brebis est bien sûr d'abord basée sur l'herbe pâturée. Trois niveaux de quantités de concentrés leur sont distribués :

-7 à $45 \mathrm{~kg}$ par brebis et par an dans les trois élevages pratiquant le plein-air et/ou n'ayant que des mises bas d'hiver, ainsi que dans les deux élevages où les brebis allaitantes sortent quotidiennement au pré pendant les agnelages d'automne et d'hiver ;

- $104 \mathrm{~kg}$ dans l'élevage où les brebis sont hivernées ensemble plus de quatre mois ;

- 50 à $82 \mathrm{~kg}$ dans les sept autres élevages où les brebis rentrent un à deux mois et demi en bergerie pour l'agnelage d'hiver, celui-ci étant complété par des mises bas d'automne.

\subsection{L'élevage des agneaux}

Les agneaux nés à l'automne sont élevés en bergerie, Les agneaux nés à partir de début janvier sont élevés à l'herbe, à une exception près. Les pratiques d'élevage des agneaux de bergerie sont peu variables alors qu'elles sont très diversifiées pour les agneaux d'herbe, sauf pour les pratiques sanitaires (rythme mensuel).

Les plus faibles quantités de concentrés distribués aux agneaux (de 0 à $20 \mathrm{~kg}$ par agneau vendu au cours de l'année) se retrouvent associées à deux types de situations : 1) production unique d'agneaux d'herbe, la complémentation est alors soit inexistante, soit limitée à la période d'allaitement, 2) production d'agneaux d'herbe et de bergerie, les agneaux d'herbe ne sont pas complémentés. Dans toutes les autres situations (complémentation au pré en été et automne ou finition en bergerie des agneaux d'herbe, avec en plus une production d'agneaux d'automne exclusivement en bergerie), cette quantité de concentrés est comprise entre 35 et $55 \mathrm{~kg}$ par agneau. 


\subsection{L'allotement des brebis}

Deux types de formules d'allotement des brebis sont identifiables.

1) Une formule simple et stable, où les lots présents à la mise à l'herbe ne sont que très peu remaniés jusqu'à la période suivante de mise bas d'hiver. Plus de la moitié des lots ont une composition inchangée entre deux hivers consécutifs. Le nombre d'opérations d'allotement au pâturage par lesquels les éleveurs modifient la composition des lots est inférieur ou égal à 2 . Les brebis agnelant en hiver sont regroupées selon leur date d'agnelage (précoces, tardives).

2) Une formule complexe : le nombre d'opérations d'allotement est compris entre 5 et 8 et moins d' $1 / 3$ des lots sont de composition stable à l'échelle de la saison. Ces opérations d'allotement ont alors pour objectif de regrouper les lots de brebis après le tarissement et/ou de préparer la reproduction (séparation des brebis taries maigres de celles en bon état corporel, constitution des lots de lutte). Les brebis sont regroupées à la mise à l'herbe surtout selon qu'elles allaitent des agneaux simples ou doubles.

\section{DIVERSITÉ DES PROJETS D'ÉLEVAGE}

La caractérisation des projets d'élevage (tableau $I$ ) a été réalisée en deux étapes, détaillées dans un article précédent [5]. La première étape vise à préciser, à partir de l'analyse des modalités des pratiques, les arbitrages entre recherche d'autonomie fourragère (recours minimal aux concentrés) et recherche d'un étalement des ventes (augmentation du pourcentage d'agneaux vendus au premier semestre). La deuxième étape aborde la simplicité de la conduite.

\subsection{Entre autonomie fourragère et demande de la filière}

Trois grands types de conduite ont pu être distingués.
1) Autonomie fourragère sinon rien $(n=2)$ : la conduite adoptée se rapproche de celle des systèmes ovins montmorillonnais traditionnels (mise bas uniquement en hiver, jamais de concentrés aux agneaux).

2) Concilier une autonomie fourragère forte et des ventes précoces $(n=3)$ : cette recherche de ventes précoces s'appuie sur des mises bas d'automne et de janvier. Dans deux élevages, la recherche d'autonomie concerne l'élevage des agneaux d'herbe (sans distribution de concentré, sauf pour les tardifs à la fin de l'automne) et l'alimentation des brebis par la sortie quotidienne systématique des mères pendant la période d'allaitement en bergerie. Le dernier cas correspond à une formule originale d'élevage de plein air : les agnelages débutent mi-décembre. Les agneaux sont complémentés durant la phase d'allaitement et de manière très sélective (les plus gros seulement) en début d'été.

Pas de recherche d'autonomie : plutôt des ventes étalées $(n=8)$ : les conduites adoptées ont trois points communs : des mises bas à l'automne, un hivernage strict des brebis agnelant en hiver et un élevage des agneaux d'herbe avec du concentré distribué après la période de sevrage. Quatre éleveurs complètent la pratique d'agnelages de contre-saison par des agnelages de début janvier et/ou une complémentation des agneaux pendant la phase d'allaitement.

\subsection{La simplicité de conduite}

Les formules d'allotement traduisent le degré de sophistication dans la gestion au cours du temps de la diversité des états des animaux qui forment le troupeau. L'allotement constitue notre indicateur de simplicité/complexité de la conduite. Les deux formules sont réparties indifféremment dans les trois types de conduite identifiées ci dessus. La formule simple est plus représentée chez les agriculteurs travaillant seuls (4 éleveurs sur 6) que dans les situations avec deux ou plus de deux travailleurs perma- 
Élevage, espace et environnement

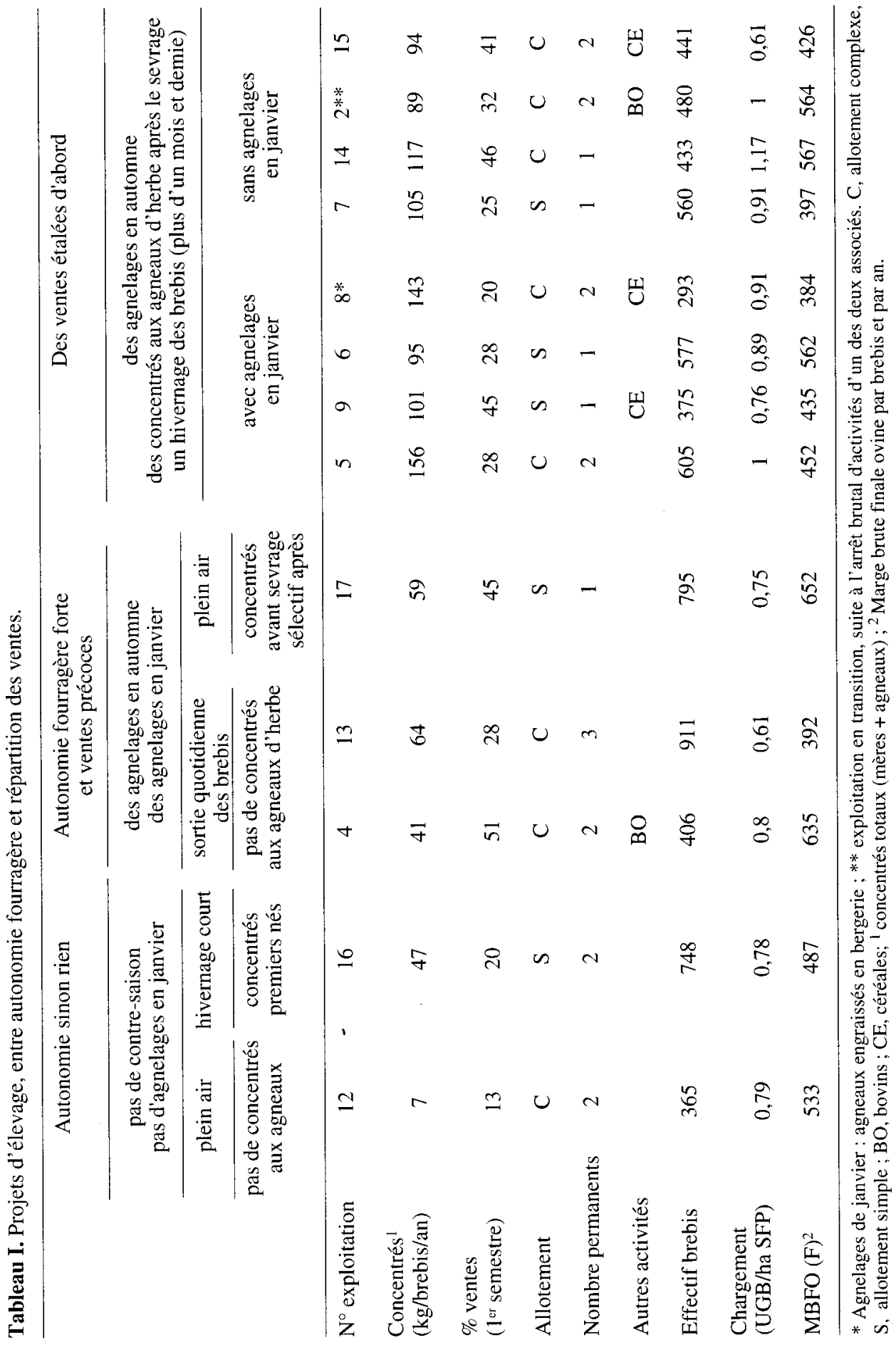


nents ( 1 sur 7). Au bout du compte, les projets d'élevage identifiés en référence aux trois éléments (autonomie, fillière, simplicité de conduite) sont très divers au sein de cet échantillon restreint.

\section{LES RÉSULTATS D'ÉLEVAGE}

\subsection{Répartition des ventes d'agneaux}

Cinq exploitants commercialisent plus de $40 \%$ de leurs agneaux au premier semestre (agneaux d'herbe de moins de 6 mois et agneaux de bergerie, hors agneaux d'herbe âgés de l'année précédente). Deux des trois élevages « autonomie + ventes précoces » en font partie, ainsi que trois élevages "des ventes étalées d'abord » (tableau I). À l'opposé, trois exploitants commercialisent moins de $20 \%$ de leur production d'agneaux jeunes au premier semestre, dont les deux « autonomie sinon rien $»$.

Le pourcentage d'agneaux d'herbe nés dans l'hiver et vendus au $1^{\text {er }}$ juillet varie de 0 à $45 \%$. Deux types de pratiques sont associées aux plus forts pourcentages de ventes précoces d'agneaux d'herbe (plus de $22 \%$ ) : i) la distribution de concentrés aux agneaux avant le sevrage, ii) la pratique d'agnelages de début janvier et ce, même en l'absence de complémentation des agneaux avant le sevrage (une exception). A l'inverse les pourcentages les plus faibles (moins de $15 \%$ ) sont associés à des mises bas débutant à partir de fin janvier sans complémentation pendant l'allaitement.

\subsection{Les résultats techniques et économiques}

La productivité numérique annuelle (nombre d'agneaux vivants par femelle de plus de 12 mois) varie de 100 à $141 \%$ (119 en moyenne). Le type de conduite (arbitrage entre autonomie et filière ; allotement simple ou complexe) n'apparaît pas comme un fac- teur de variation des performances de reproduction (fertilité, prolificité des brebis et mortalité des agneaux). Les quantités totales de concentrés distribués au troupeau varient de 7 à $157 \mathrm{~kg}$ par brebis et par an $(86 \mathrm{~kg}$ en moyenne). La marge brute finale ovine (MBFO) varie de 384 à 654 F/brebis de plus de 12 mois dans l'échantillon pour une moyenne de $498 \mathrm{~F}$.

La distinction entre les deux premiers groupes (une composante « recherche d'autonomie » exclusive ou non) et le troisième groupe est assez nette sur les quantités de concentrés. En revanche, dans chacun des groupes, les niveaux de MBFO sont variables (tableau $I$ ). Chaque type de projet d'élevage semble avoir un sens du point de vue de l'économie de la production. Mais la variabilité intra-type de MBFO traduit la plus ou moins bonne maîtrise des autres composantes influençant cette marge brute: productivité numérique, classement des carcasses et charges de la SFP [3].

\section{DISCUSSION}

Notre étude, basée sur un petit nombre d'exploitations, n'a pas de prétention à la représentativité des cas étudiés. Elle confirme la diversité des projets d'élevage associé au caractère extensif, cette diversité pouvant être requalifiée en terme d'enjeux autour de la gestion de la surface fourragère différents d'une part, et de pratiques et techniques mobilisables pour cette gestion d'autre part.

Les opportunités de modification rapide des périodes de mises bas apparaissent comme un puissant levier d'adaptation aux changements. Un choix donné de combinaisons de dates de lutte et de mode d'élevage des agneaux a pour conséquence une répartition des périodes de forts besoins du troupeau dans le temps [14] : il définit un profil particulier d'enchaînement d'enjeux saisonniers vis-à-vis de la gestion de la surface fourragère [9]. Par exemple, dans le 
projet « autonomie et ventes précoces au printemps », l'hiver est une période critique importante dans le calendrier du pâturage : les brebis agnelant en janvier doivent trouver une bonne partie de leur ration à l'herbe (économie de concentrés) et assurer le bon démarrage des agneaux d'herbe (les vendre rapidement). La création de ressources sur pied pour assurer le bon déroulement de cette période est alors un objectif essentiel. On dispose de peu de références sur le pâturage des brebis et l'élevage des agneaux dans ces conditions [8], mais sa réussite dépend vraisemblablement, en conditions herbagères, des capacités d'anticipation des éleveurs dans la gestion du pâturage (mis en défens dès l'automne) et dans la conduite de la reproduction (ajustement de l'effectif de brebis concerné par cette période critique [11]).

La pratique d'une lutte complémentaire de contre-saison, si elle répond aux incitations de la filière, permet également d'avoir à chaque instant des brebis à niveau de besoins alimentaires différents. Cela constitue un avantage pour la gestion de la diversité des ressources pâturées, comme en témoignent les périodes de reproduction adoptées dans les expérimentations sur l'élevage ovin extensif $[2,7]$. On comprend que ces choix de répartition apparaissent stratégiques, pour ce qu'ils traduisent des projets d'élevage, mais aussi du fait de leurs conséquences sur les techniques appropriées de conduite, en particulier du pâturage.

Le découpage du troupeau en lots quasiment indépendants sur la campagne en vue de simplifier les raisonnements de conduite a été observé dans d'autres situations contraignantes en travail notamment en élevage bovin allaitant [13]. Limiter les opérations d'allotement, c'est se priver d'un outil pour l'ajustement de la diversité des besoins alimentaires des animaux à celles des ressources fourragères : l'allotement est la composante structurante de l'organisation des circuits de pâturage [6]. Une conduite basée sur le principe d'une stabilité des lots à l'échelle de la saison entraîne la nécessité de recourir à d'autres outils de régulation de la pousse et de la qualité de l'herbe, tels que le gyrobroyeur.

\section{CONCLUSION}

L'extensif constitue un contexte dans lequel les éleveurs raisonnent des projets d'élevage variés, dépendants d'arbitrage entre des contraintes internes à l'exploitation (dimension, main d'œuvre, aptitudes agronomiques et externes: PAC, mesures agri-environnementales, filières). Ces arbitrages ne leur sont pas totalement spécifiques : les conduites de la reproduction (avancement des agnelages, contre-saison) se distinguent peu de celles adoptées dans d'autres systèmes de production. En revanche l'opportunité de développer des projets d'élevage basés essentiellement sur l'herbe est facilité en situation extensive par un meilleur ajustement de la charge animale aux périodes difficiles du calendrier fourrager (hiver et été). L'économie de charges d'alimentation peut porter non seulement sur l'élevage des agneaux d'herbe [20] mais aussi sur l'alimentation des brebis.

La diversité des projets d'élevage a une double conséquence sur la gestion des systèmes extensifs : la variété des choix de périodes de mise bas entraîne de grandes différences dans les articulations entre périodes de forts besoins du troupeau et dynamique des ressources fourragères ouvrant notamment la question de la gestion de la période hivernale au pâturage pour des animaux à besoins élevés. De plus, les pratiques et techniques utilisées pour gérer le territoire ne seront pas identiques selon les projets. La diversité des besoins des animaux à un instant donné, permise par des mises bas à périodes différentes n'est pas donnée dans un système extensif, les formes d'adaptation de l'organisation du pâturage par de fréquents réallotements des troupeaux non plus. Ces considérations militent pour l'intégration de la connaissance de la diver- 
sité et du contenu des projets d'élevage dans la formulation de questions scientifiques et techniques sur l'extensif.

\section{REMERCIEMENTS}

Ce travail a été réalisé avec la participation de J. Willaert (Inra-SAPF), V. Bellet (CRA, Poitou-Charentes), G. Liénard (Inra-LEE), O. Pagnot (CA Vienne), J. Lamoureux (CA Vienne).

\section{RÉFÉRENCES}

[1] AFPF, L'extensification en production fourragère, Fourrages ( ${ }^{\circ}$ Spécial), 1992, 192 p.

[2] Chabosseau J.M., Laignel G., Lamoureux J., Souille C., Bergeron C., Staub A., Étude de Ja conduite et des performances de deux unités expérimentales chargées à 5 et 8 brebis/ha, Doc Inra/Lepa, Montmorillon/Chambre d'Agriculture de la Vienne, 1996, $143 \mathrm{p}$.

[3] Chabosseau J.M., Dedieu B., La prairie temporaire et les interventions mécaniques dans les systèmes fourragers extensifs : des exemples en Montmorillonnais, Fourrages, 151 (1997) 35 $1-372$.

[4] Cifar, L'extensification, une forme de modernisation, Maf-Derf et Comité national extensification-diversification, $1990,192 \mathrm{p}$.

[5] Dedieu B., Chabosseau J.M., Benoit M., Laignel G., L'élevage ovin extensif du Montmorillonnais entre recherche d'autonomie, exigences des filières et simplicité de conduite, Inra Prod. Anim. 10 (1997) 207-218.

[6] Dedieu B., Chabanet G., Josien E., Bécherel F., Organisation du pâturage et situations contraignantes en travail : démarche d'étude et exemples en élevage bovin viande, Fourrages 149 (1997) 21-36.

[7] De Montard F.X., Louault F., Thériez M., Brelurut A., Pailleux J.Y., Benoit M., Liénard G., Recherches sur les techniques de production extensive en élevage ovin. Quatre années d'expérimentation au domaine de Redon (1988-1991), Doc. de travail Inra centre de Clermont-Ferrand Theix, 1992, 28 p. + ann.

[8] Gibon A., Pratiques d'éleveurs et résultats d'élevage dans les Pyrennées centrales, thèse $I$ na $P G$, 1981, $106 \mathrm{p}$.

[9] Girard N., Modéliser une représentation d'experts dans le champ de la gestion de l'exploitation agricole, thèse Université Claude Bernard-Lyon I, Inra SAD/IE, 1995, 234 p. + ann.
[10] Grenet N., Dedieu B., Dozias D., Hardy A., Micol D., Research on extensification of beef and sheepmeat production in France: description, preliminary results and prospects, in : Keane M.G., Pflimilin A. (éds.), Extensification of beef and sheep production on grasslands, Proceeding of Concerted Action AIR 3-CT930947, Paris Workshop, 22-24 nov. 1995, 1996, $27-46$.

[11] Hill Farming Research Organisation (HFRO), Science and Hill Farming: twenty years of work at the HFRO 1954-1979 (published by the HFRO on the occasion of their jubilee, Sept 79), Edinburgh, 1979, 184 p.

[12] Ingrand S., Dedieu B., Chassaing C., Josien E., Étude des pratiques d'allotement dans les exploitations d'élevage. Proposition d'une méthode et illustration en élevage bovin extensif, Inra Et. Rech. Syst. Agr. Dev. 27 (1993) 237-302.

[13] Ingrand S., Dedieu B., Diversité des formules d'allotement en élevage bovin viande. Le cas d'exploitation du Limousin, Inra Prod. Anim. 9 (1996) 189-199.

[14] Jarrige R., Utilisation des pâturages des milieux défavorisés : essai de conclusion, in : Molénat $\mathrm{G}$., Jarrige R. (éds.), Utilisation par les ruminants des pâturages d'altitude et des parcours méditerranéens, Inra X Journées du Grenier de Theix (1979) 541-565.

[15] Journet M., Extensification des herbivores, Bull. Tech. Inf. Min. Agric., 25 (1996) 14-24.

[16] Landais E., Deffontaines J.P., Les pratiques des agriculteurs. Point de vue sur un nouveau courant de la recherche agronomique. Études Rurales 109 (1989) 125-158.

[17] · Landais E., Gilibert J., Recherche sur l'extensification de l'élevage. Éléments de réflexion tirés d'une approche systémique, Inra-Sad VersaillesDijon-Mirecourt, document de travail, 1991, $55 \mathrm{p}$.

[18] Micol D., Dedieu B., Agabriel J., Béranger C., Adaptation de la production de viande bovine aux systèmes extensifs d'élevage, Fourrages, 149 (1997) 3-20

[19] Pavie J., Pflimlin A., Réseaux d'élevage extensifs bovins et ovins. Bilan des premières années de suivi 1990-1994. Acquis, manques, propositions, Doc. Institut de l'élevage/Inra Sad/Enita Clermont-Ferrand/Chambres d'Agricultures, 1995, $104 \mathrm{p}$.

[20] Prache S., Thériez M., Béchet G., Complémentation des agneaux au pâturage pendant la phase d'allaitement, Inra Prod. Anim. 5 (1992) 137-148. 